\title{
Das „Ambulante Gruppenprogramm zum kontrollierten Trinken (AkT)“
}

\author{
Joachim Körkel $^{1}$ \\ Birgit Schellberg ${ }^{2}$ \\ Karin Haberacker ${ }^{3}$ \\ Wilfried Langguth ${ }^{2}$ \\ Birgit $\mathrm{Neu}^{2}$
}

\author{
The Outpatient Group Treatment Program for Controlled Drinking
}

\section{Zusammenfassung}

Das „Ambulante Gruppenprogramm zum kontrollierten Trinken (AkT)“ ist ein seit 1999 erprobtes, manualisiertes verhaltenstherapeutisches und lösungsorientiertes Programm zur Vermittlung von Kompetenzen zur Selbstkontrolle des Trinkverhaltens. Es richtet sich an Menschen mit akuten Alkoholproblemen unterschiedlichen Schweregrades und (zumindest anfänglicher) Ablehnung des Abstinenzziels. Das Akt umfasst 1-2 diagnostische Einzelsitzungen und 10 wöchentliche, inhaltlich und didaktisch standardisierte Gruppentermine (max. 14 Teilnehmer) a $2^{1 / 4}$ Stunden, geleitet von ein bis zwei im AkT fortgebildeten Suchtfachkräften. Die Ergebnisse für die ersten N = 58 AkT-Teilnehmer (Durchschnitt 48 Jahre, 33\% Frauen, 67\% alkoholabhängig [ICD-10]) zeigen eine hohe Zufriedenheit mit den 10 Gruppensitzungen und eine durchschnittliche wöchentliche Trinkmengenreduktion von mehr als 50\% (signifikant mehr als in einer Wartekontrollgruppe, $\mathrm{p}<.001$ ), die auch nach einem Jahr noch nachweisbar ist. Etwa 10\% der Teilnehmer leben abstinent, $47 \%$ befinden sich in Weiterbehandlung (mit dem Ziel der Abstinenz oder des kontrollierten Trinkens). Weitere Programmentwicklungen werden aufgezeigt.

\section{Schllïsselwörter}

Kontrolliertes Trinken · Selbstkontrollprogramm · Alkoholprobleme

\section{Abstract}

The „Outpatient Group Treatment Program for Controlled Drinking“ (in German „Ambulantes Gruppenprogramm zum kontrollierten Trinken", AkT) is a Behavioral Self-Control Training with the goal of controlled drinking (max. 14 participants) run in Germany since 1999. It encompasses $1-2$ pre-assessment sessions (individual format) and 10 structured group sessions (one session per week, $2^{1} / 4$ hours each) delivered by $1-2$ addiction specialists who are trained to use a manual for pre-treatment assessment and execution of treatment. The AkT addresses people with different degrees of alcohol problems. Till now five AkT groups with 58 Ss (mean age 48 years, $33 \%$ women, $67 \%$ alcohol dependent [(ICD-10]) have been conducted and evaluated highly positive by participants. Reduction in weekly standard drinks from beginning to end of $\mathrm{AkT}$ is more than 50\% (significantly higher than in a waiting list control group, $\mathrm{p}<.001$ ) and stays at this level at 1-year follow-up. $10 \%$ of Ss are abstinent at followup and $47 \%$ are in further treatment (for abstinence or controlled drinking). Latest program developments are reported.

\section{Key words}

Controlled Drinking $\cdot$ Behavioral Self-Control Training $\cdot$ Alcohol Problems
Das AkT ist ein ambulantes, 10 wöchentliche Sitzungen zu je $2^{1 / 4}$ Zeitstunden umfassendes strukturiertes Gruppenprogramm, in dem sich die Teilnehmer - bis zu 14 Personen - in zieloffener Weise für kontrolliertes Trinken oder Abstinenz entscheiden können. „(Selbst)kontrolliertes Trinken“ bedeutet hier, dass der Konsument sein Trinkverhalten an zuvor von ihm festgelegten Trinkregeln - vor allem Trinkmenge und -häufigkeit betreffend - ausrichtet. Das AkT versteht sich als Ergänzung zu den vorhan-

Institutsangaben

${ }^{1}$ Evangelische Fachhochschule Nürnberg, Fachbereich Sozialwesen

2 Psychosoziale Beratungs- und Behandlungsstelle für Suchtkranke des Caritasverbandes Nürnberg

${ }^{3}$ Psychologisches Institut der Universität Heidelberg

Korrespondenzadresse

Prof. Dr. Joachim Körkel · Ev. Fachhochschule Nürnberg, Fachbereich Sozialwesen · Bärenschanzstraße 4 . 90429 Nürnberg · E-mail: joachim.koerkel@evfh-nuernberg.de 
denen Abstinenzangeboten. Es wurde erstmals im Oktober 1999 an der Psychosozialen Beratungs- und Behandlungsstelle für Suchtkranke des Caritasverbandes Nürnberg durchgeführt [1,2]. Seit April 2001 werden Trainerkurse zum AkT angebotenen [3]. Auf diesem Weg haben sich inzwischen über 140 Suchtfachkräfte aus Deutschland und der Schweiz zur eigenständigen Durchführung von AkT-Kursen qualifiziert. Das AkT wird inzwischen in verschiedenen Städten Deutschlands und der Schweiz angeboten, integriert in die Angebotspalette von Beratungsstellen und Praxen (vgl. www.kontrolliertes-trinken.de). Für die Teilnahme am AkT wird eine vom lokalen Anbieter frei wählbare Gebühr erhoben, zum Teil ist sie kostenfrei (wie in Nürnberg).

\section{Zielgruppen und Ziele des AkT}

Das AkT wendet sich an Menschen, die vier Voraussetzungen erfüllen („Indikation“):

- Ihr Alkoholkonsum hat ein riskantes bzw. bereits schädigendes Ausmaß angenommen.

- Sie haben den Wunsch, ihren Alkoholkonsum zu reduzieren.

- Ein einfaches „Herunterschrauben“ des Alkoholkonsums ist ihnen nicht möglich.

- Abstinenz ist für sie kein zur Zeit gewünschtes oder realistisches Ziel.

Mit dem AkT werden die folgenden Zielsetzungen verbunden:

- Personen zu einer Teilnahme zu motivieren, die sich durch Hilfsangebote mit ausschließlicher Abstinenzvorgabe (und oft langer oder unklarer Behandlungsdauer) nicht oder nicht mehr angesprochen fühlen;

- konkrete Hilfestellungen zur Konsumreduktion bzw. bei Entwicklung von Abstinenzmotivation zum Erreichen der Abstinenz zu geben;

- Beeinträchtigungen, die durch überhöhten Alkoholkonsum entstanden sind, abzubauen;

- Teilnehmer, denen eine Trinkmengenreduktion nicht zufrieden stellend gelingt, in abstinenzorientierte Suchthilfeangebote (z.B. ambulante Beratung, ambulante oder stationäre Entwöhnung) zu vermitteln.

Kontraindikationen lassen sich in Bezug auf das AkT in „absolute“ und "bedingte“ unterscheiden:

- Eine absolute Kontraindikation besteht in bereits erreichter Abstinenz oder einem festen Entschluss zu dauerhafter Abstinenz.

- Bedingte Kontraindikationen liegen vor a) bei einem schweren Alkoholproblem (wie etwa Alkoholabhängigkeit) und b) bei auch durch geringe Alkoholmengen absehbaren negativen Konsumfolgen (wie im Falle von Schwangerschaft/Stillzeit, alkoholsensibler Medikation oder körperlichen Vorschädigungen). „Bedingt“ sind diese Kontraindikationen zu nennen, weil in diesen Fällen die Alkoholabstinenz zwar Ziel erster Wahl ist, kontrolliertes Trinken aber trotzdem geboten ist, wenn der Konsument nicht in der Lage ist, Abstinenz anzustreben oder einzuhalten, und wenn er das Abstinenzziel ausdrücklich ablehnt und statt dessen eine Konsumreduktion wünscht.

- Behandlungsbedürftige psychiatrische Begleiterkrankungen (z.B. schwere Depression, Borderline-Störung oder Psychose), zusätzlicher Suchtmittelkonsum (z.B. Cannabis), massive so- ziale Desintegration (z.B. Wohnsitzlosigkeit) oder andere Beeinträchtigungen der „Gruppenfähigkeit“ (z.B. extrem schlechtes Hörvermögen; bedeutsamer kognitiver Abbau) stellen zwar keine Kontraindikationen für kontrolliertes Trinken dar. Sie können es aber geraten erscheinen lassen, statt des Gruppenprogramms das „Einzelprogramm zum kontrollierten Trinken“ (EkT) [4] zur Anwendung zu bringen.

\section{Screening und Diagnostik im AkT}

Dem AkT vorgeschaltet finden 1-3 diagnostische Einzelsitzungen statt. Es kann bei einer einzigen Sitzung bleiben, a) wenn sich bereits aus dem Erstkontakt eine eindeutige Kontraindikation für die AkT-Teilnahme ergibt oder b) wenn man den Klienten einige der diagnostischen Verfahren selbstständig zu Hause bearbeiten lassen kann und möchte.

In der ersten Diagnostiksitzung, dem Screening, werden anhand des „Diagnostik-Manuals“ [5] das aktuelle Trinkverhalten, das Ausmaß körperlicher Entzugserscheinungen, eventuelle psychische Begleiterkrankungen und Konsum weiterer psychoaktiver Substanzen sowie der Grad der sozialen Integration erhoben. Wenn sich aus diesen Informationen nicht bereits eine Kontraindikation für das AkT ergibt, wird in dieser Sitzung noch der Auftrag an den zukünftigen AkT-Teilnehmer weitergegeben, bis zum nächsten Diagnostiktermin aktuelle Ultraschallbefunde (Abdomen) und Laborwerte (Gamma-GT, GOT, GPT, Bilirubin, Quick, Albumin, kleines Blutbild, BSG) einzuholen und eine körperliche Untersuchung vornehmen zu lassen (mit vorgefertigtem Anschreiben an den behandelnden Arzt).

Beim zweiten und ggf. dritten Diagnostiktermin werden die alkoholbezogene (ICD-10, AUDIT, NASS [dt. Übersetzung des SADQ], KAZ-35 [modifizierte Version für kontrolliertes Trinken]) und weitere psychiatrische Symptomatik (DIA-X-SSQ) vertiefend abgeklärt sowie soziodemographische, suchtbiographische und soziale Basisdaten (gekürzte Form des EuropASI) erhoben.

\section{Grundllagen und Aufbau des AkT}

Das AkT mit seinen 10 Sitzungen gründet auf mehreren Pfeilern:

- Die wissenschaftliche Basis bilden die Erkenntnisse der Forschung zum kontrollierten Trinken [6,7,8,9; www.kontrolliertes-trinken.de] und zum „Stufen-der-Veränderung-Modell“ [10].

- Der Aufbau des Programms ist verhaltenstherapeutisch orientiert (klare Programmstruktur, Transparenz des Vorgehens, psychoedukative Programmelemente, erfahrungsorientiertes Neulernen, „Hausaufgaben“, Selbstkontrollmodell).

- Die individuumsbezogenen Interventionen der Gruppenleitung orientieren sich an den Prinzipien und Methoden der „motivierenden Gesprächsführung“ (z.B. aktives Zuhören, "geschmeidiger“ Umgang mit Widerstand usw. [11]) sowie lösungsorientierter Therapie (Herausarbeitung der Fortschritte der Teilnehmer sowie der Kompetenzen, die die Fortschritte begünstigt haben, anstatt in „Problemtrancen“ zu verfallen ein äußerst aufbauendes, motivierendes Vorgehen für die Teilnehmer! [12]). 
- Der Tatsache eines Gruppenprogramms wird Rechnung getragen durch die Berücksichtigung gruppenpädagogischer Prinzipien (z. B. Einführung von Ruth Cohns TZI-Regeln) und einer moderner Erwachsenenbildung genügenden Didaktik (Nutzung von aktivierenden Gruppenmethoden, Visualisierungen, Overhead-, Flip-Chart- und Metaplanmethodik usw.).

- Last but not least: Das Menschenbild ist humanistisch-psychologisch orientiert (Respektierung und Förderung der Klientenautonomie als übergeordnete ethische Maxime, z.B. bei Zielentscheidungen; Berücksichtigung der aktuellen Befindlichkeiten und Wünsche der Teilnehmer [„Hier-und-JetztPrinzip“], statt „kontaktarmer Stoffabarbeitung“; Zuversicht in die Klienten, dass diese das ihnen gemäße Veränderungstempo selbst herausfinden usw.).

Die 10 Gruppenabende, die von 1-2 für das AkT fortgebildeten Suchtfachkräften geleitet werden [3], gestalten sich wie folgt:

Im ersten Teil der Sitzung äußert sich jeder Teilnehmer nach einem kurzen Befindensblitzlicht zu seinem Umgang mit Alkohol und zu weiteren wichtigen Ereignissen während der letzten $7 \mathrm{Ta}$ ge. Weiterhin nimmt er für die bis zum nächsten Gruppentermin folgenden 7 Tage eine Zielplanung anhand von 3 Parametern vor (Anzahl abstinenter Tage; maximale Trinkmenge an Trinktagen; maximale wöchentliche Konsummenge). Die Gruppenleitung fokussiert und präzisiert die Wochenerfolge und gibt bei Bedarf Unterstützung bei der weiteren Zielplanung.

Im zweiten Teil der Sitzung werden festgelegte Inhalte vermittelt bzw. gemeinsam erarbeitet. Dazu gehören: Grundinformationen über Alkohol (u.a. Ethanolgehalt der gebräuchlichsten Alkoholika, Definition einer Standard[getränke]einheit, Bestimmung der Blutalkoholkonzentration), Handhabung des Trink-Tagebuchs (das über das ganze Programm hinweg zu führen ist), persönliche Gründe für eine Konsumreduktion („Motivatoren“), Möglichkeiten der Trinkzielfestlegung für jeweils eine Woche, Identifizierung von Risikosituationen für überhöhten Alkoholgebrauch, Strategien zur Konsumbegrenzung, Umgang mit Rückschlägen und Entwicklung von Alternativen zum Alkoholkonsum (Bewältigung von Belastungen und Freizeitgestaltung). In diesem zweiten Teil der Gruppensitzungen wechseln sich in der Didaktik visuell unterstützte Kurzbeiträge der Gruppenleitung (z.B. zu Grundinformationen über Alkohol), Einzelarbeiten der Teilnehmer (z.B. Bearbeitung von Checklisten zu Risikosituationen), Kleingruppenarbeiten (z. B. Erarbeitung von Kontrollstrategien), Rollenspiele (z. B. zum Nein-Sagen bei Trinkangeboten), der Austausch in der Gesamtgruppe und die Besprechung von Informationsblättern ab.

Für jede einzelne der 10 AkT-Sitzungen steht ausgebildeten AkTTrainern das ausführliche „Trainer-Manual“ zur Verfügung, in dem der zeitliche und inhaltliche Ablauf mit allen didaktischen Anregungen minutiös ausgearbeitet ist, auf mögliche Durchführungsprobleme präventiv vorbereitet wird und alle Informations- und Arbeitsblätter enthalten sind [13]. Die Teilnehmer erhalten ein eigenes „Teilnehmer-Handbuch“ mit Sitzungsübersichten, Arbeits- und Informationsbögen sowie weiteren Unterlagen [14].

\section{Qualitätssicherung}

In das AkT ist eine systematische Qualitätssicherung implementiert.

Im Sinne der Strukturqualität ist die Diagnostik standardisiert, d.h. es findet eine Batterie national und international verbreiteter Diagnostikinstrumente zur Erhebung des bio-psycho-sozialen Status der AkT-Teilnehmer Anwendung („Diagnostik-Manual“, s.o.). Das umfangreiche AkT-Trainermanual mit seinen Materialien und exakten Durchführungshinweisen und Zeitangaben für die einzelnen Abschnitte jeder Sitzung bildet die Grundlage für eine standardisierte, aktuellen fachlichen Standards genügende Gruppendurchführung. Die Gruppenteilnehmer werden mit dem „Teilnehmer-Handbuch“ ausgestattet, wodurch gewährleistet ist, dass sie über alle für die einzelnen Sitzungen notwendigen Unterlagen verfügen und mit diesen nach dem AkT weiterarbeiten können. Eine hohe Qualität des AkT wird weiterhin dadurch gefördert, dass zu den Trainerschulungen nur Suchtfachleute zugelassen werden, das Training umfassend angelegt ist (von der Diagnostikschulung über die Einübung aller 10 Sitzungen bis zur Heranführung an die Anwendung der ausgearbeiteten Katamnesebögen), nur ausgebildete AkT-Trainer das „Trainer-Handbuch“ erwerben können und ausgebildete AkT-Trainer in „senior trainings“ Möglichkeiten zum „Qualitäts-Check-up“ und zur Qualitätsoptimierung erhalten [15].

Die Prozess- und Ergebnisqualität des AkT werden systematisch geprüft u.a. durch anonym auszufüllende Stundenbeurteilungsbögen am Ende jeder Sitzung (für Teilnehmer und Trainer), Teilnehmer-Abschlussbefragungen (summative Programmevaluation) und Katamnesen (Laborbefunde und Befragungen zum Trinkverhalten sowie körpermedizinischen, psychischen und sozialen Status nach 6,12 und 24 Monaten).

Schließlich werden auch die AkT-Trainerseminare mittels eines standardisierten Fragebogens durch die Teilnehmer evaluiert (u.a. Zufriedenheit mit 11 Seminaraspekten, wie „Kompetenzzuwachs“, „Praxisnähe“, „schriftliche Unterlagen“ etc.). Stellvertretend für die Zufriedenheit mit den 11 Seminaraspekten steht das positive Gesamturteil zu jedem der 6 Trainingskurse: Es liegt auf einer Skala von -3 („sehr schlecht“) bis + 3 („sehr gut“) nie unter $+2(\mathrm{n}=107$ Trainingsteilnehmer $)$.

\section{Ergebnisse zum AkT}

\section{AkT-Teilnehmer}

Bislang liegen Daten zu den ersten fünf von uns zwischen Oktober 1999 bis Juli 2001 in Nürnberg durchgeführten AkT-Gruppen vor. Einige Interessenten wurden in dieser Zeit nicht in das Programm aufgenommen (u. a. wegen Schwerhörigkeit, starker Verwahrlosung und hirnorganischem Abbau, starker Angststörung, ausgeprägter Depression und THC-Gebrauch). Von den 58 teilnehmenden Personen waren 19 Frauen und 39 Männer, alle über $30 \mathrm{Jahr}$ alt (Durchschnitt 48 Jahre), mehr als die Hälfte verheiratet. Mehr als $75 \%$ wiesen mindestens die mittlere Reife als Schulabschluss auf, ebenso viele waren arbeitstätig (die Hälfte im Angestelltenverhältnis [z.B. Altenpflegerin], ein Viertel als Selbstständige [z. B. Werbefachmann] und ein Fünftel als Beamte 
[z. B. Lehrer]). Zwei Drittel der Teilnehmer (67\%) hatten vor dem AkT noch keinerlei Kontakte zum Suchthilfesystem gehabt.

Die durchschnittliche Trinkmenge der AkT-Teilnehmer (hier $\mathrm{n}=52$ ) betrug unmittelbar vor AkT-Beginn (zum Teil bereits reduziert durch das Wissen um den baldigen Programmbeginn) $28 \mathrm{SE}$, bei erheblicher Variation um diesen mittleren Wert ( $S D=15,57$; Spannbreite 0 - 70 SE; Abb. 1).

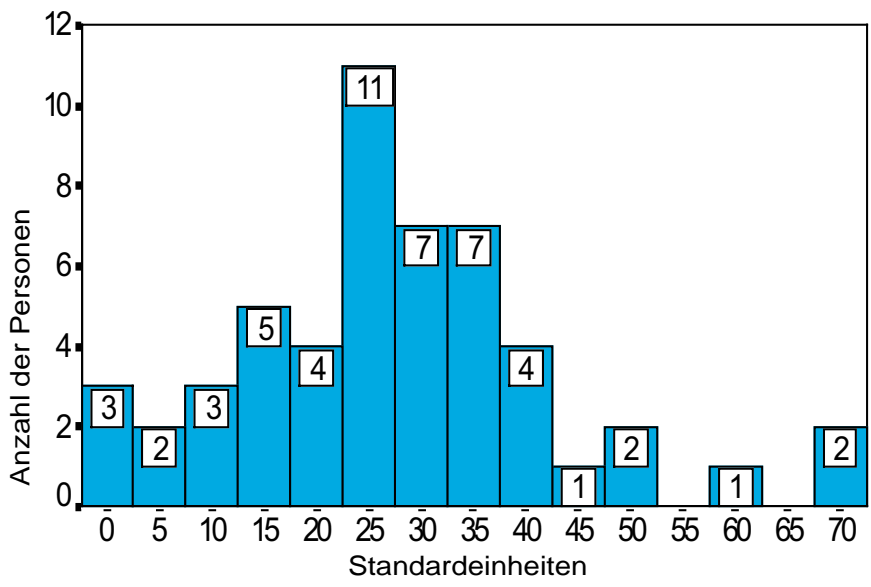

Abb. 1 Trinkmenge pro Woche vor AkT-Beginn in Standardeinheiten [SE] (1 SE= 20 g Alkohol; $n=52$ )

Zwei Drittel der Teilnehmer (66\%) waren als alkoholabhängig einzustufen (mindestens 3 ICD-10-Kriterien erfüllt; Abb. 2). Bei jedem vierten AkT-Teilnehmer (24\%) lag darüber hinaus Nikotinabhängigkeit vor (20 oder mehr Zigaretten/Tag).

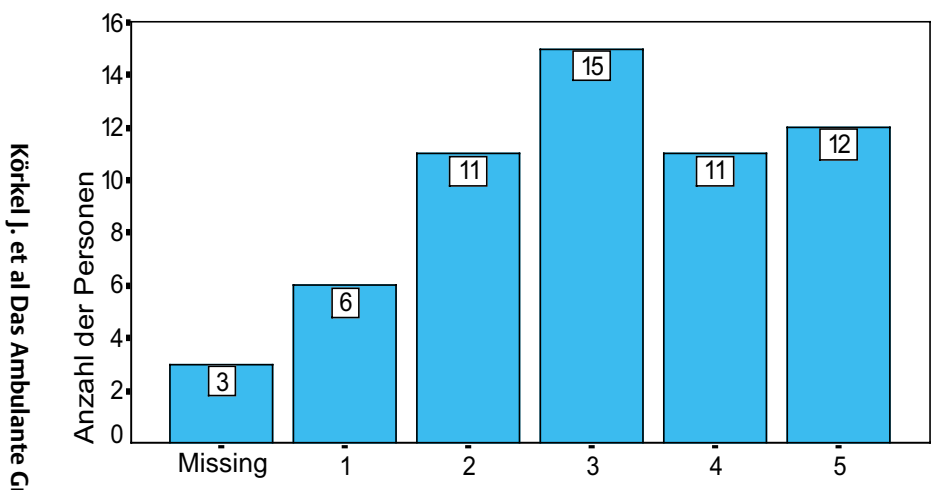

Abb. 2 Anzahl erfüllter ICD-Kriterien bei den Akt-Teilnehmern ( $n=58$ )

Jeder zweite AkT-Teilnehmer hatte bereits alkoholbedingte medizinische Folgeprobleme erfahren (z. B. 33\% eine Fettleber, 3\% eine periphere Polyneuropathie).

Neun Personen beendeten das AkT vorzeitig, zum Teil aufgrund unserer Empfehlung zur Aufnahme einer weitergehenden Behandlung (so begannen drei Personen eine Entgiftungs- und/ oder Entwöhnungsbehandlung).

\section{Konsumveränderungen im Laufe der AkT-Teilnahme (Prä-Post-Vergleich)}

Zur Abbildung von im Laufe der AkT-Teilnahme eingetretenen Veränderungen wurde das Trinkverhalten der Teilnehmer der ersten drei AkT-Gruppen („Behandlungsgruppen“; n=34) mit dem Trinkverhalten derjenigen Personen verglichen, die während der Behandlungszeit der ersten AkT-Gruppe $3^{1 / 2}$ Monate auf den Beginn ihrer Gruppe warteten („Wartekontrollgruppe“; $\mathrm{n}=12$ ). Der Vergleich erbringt folgendes:

- In den Behandlungsgruppen reduzierte sich der Alkoholkonsum im Laufe des AkT um durchschnittlich 14,8 SE pro Woche und damit signifikant stärker als in der Wartekontrollgruppe, in der die Reduktion nur 0,5 SE betrug, $\mathrm{t}(44)=3,76, \mathrm{p}<.001$.

- Gleichzeitig stieg in den Behandlungsgruppen die Anzahl der pro Woche abstinent verbrachten Tage um 1,7 Tage, in der Wartekontrollgruppe nur um 0,2 Tage an, $\mathrm{t}(44)=2,56, \mathrm{p}<.05$.

- Weitere Vergleiche belegen die positive Wirkung des AkT auch in biologischen Alkoholmarkern [16].

- Die vor dem AkT erhobene Zuversicht, in 35 verschiedenen Risikosituationen dem Zuviel-Trinken ( $=$ mehr trinken als gemäß Trinkplan vorgesehen) widerstehen zu können (KAZ-35, Version für kontrolliertes Trinken) erwies sich als prädiktiv: Je höher das Anfangszutrauen in die eigene Fähigkeit, in Trinkverführungssituationen stand zu halten, ausfiel, desto mehr abstinente Tage pro Woche waren unmittelbar nach Abschluss des AkT zu verbuchen, $R=.36, p<.05$. Noch stärker fiel dieser Zusammenhang zwischen dem Anfangszutrauen, unangenehme Gefühle ohne unkontrolliertes Trinken bewältigen zu können, und der Anzahl abstinent verbrachter Tage nach dem AkT aus, $\mathrm{R}=.73, \mathrm{p}<.01$.

Auch die Tatsache, dass alle Teilnehmer zu weitgehend allen Terminen anwesend waren und in den am Ende jeder Sitzung ausgegebenen „Stundenbeurteilungsbögen“ (6 Items, z.B. „Heute habe ich den Eindruck, dass ich bei der Bewältigung meines Alkoholproblems vorangekommen bin“) hohe Zufriedenheitswerte angaben, spricht für den guten Anklang, den das Programm gefunden hat (vgl. Abb. 3). Beispielsweise lag das summative Zufriedenheitsurteil der Teilnehmer („Insgesamt betrachtet, fand ich die heutige Gruppensitzung ...") in keiner der 10 Sitzungen unter 4 ( $0=$, ,ehr schlecht“", $5=$ „, sehr gut“). Auch unser Eindruck, dass die AkT-Teilnehmer persönlich sehr engagiert „bei der Sache waren“, „Klientenwiderstand“ (leugnen, bagatellisieren usw.) kaum aufgetaucht ist und die Suchtarbeit im Rahmen des AkT eine neue Leichtigkeit erfahren hat, sollten nicht unerwähnt bleiben.

\section{Katamnestische Ergebnisse}

Von 11 AkT-Teilnehmern liegen bislang komplette Messwerte bis zu einem Jahr nach AkT vor. Sie demonstrieren:

- Im Laufe des AkT reduzierte sich die an Trinktagen konsumierte Alkoholmenge („Drinks per Drinking Day“, DDD) deutlich und nahm im Folgejahr weiter ab (Abb. 4). Der DDDWert nach einem Jahr (2,1 SE) lag signifikant unter dem vor AkT-Beginn (4,8 SE), $\mathrm{t}(10)=3,70, \mathrm{p}<.01$.

- Der im Laufe des AkT markant gesunkene Wochenkonsum erhöhte sich in den ersten sechs Monaten nach AkT um 2,4 SE und sank im Laufe der kommenden sechs Monate exakt auf das Niveau zu Ende der Behandlung ab (12,9 SE; Abb. 5). Die Mittelwertsdifferenz zwischen der wöchentlichen Konsum- 
menge zu Behandlungsbeginn und $1 \mathrm{Jahr}$ nach AkT-Ende ist signifikant, $\mathrm{t}(10)=4,22, \mathrm{p}<.01$.

- Ein ähnlicher Verlauf bildet sich bei der Betrachtung der abstinenten Tage pro Woche ab (Abb. 6). Hier sank der Mittelwert von 2,7 Tagen unmittelbar nach Behandlungsende auf 2,2 Tage nach 6 Monaten ab, stieg aber in den kommenden 6 Monaten wieder auf 2,7 Tage an. Auch hier unterscheiden sich Ausgangs- und 1-Jahres-Wert bedeutsam, t(10) = 3,07, p <.05 .

\section{Weiterbehandlung}

Nahezu jeder zweite der bisherigen 58 Nürnberger AkT-Teilnehmer $(47 \%)$ nahm nach dem AkT irgend eine Art von weiterer suchtspezifischer Hilfe in Anspruch, u.a. Selbsthilfegruppenbesuche (14\%), fortlaufende Beratung (10\%), stationäre Entwöhnungsbehandlung (3\%) oder mehrere Behandlungsangebote

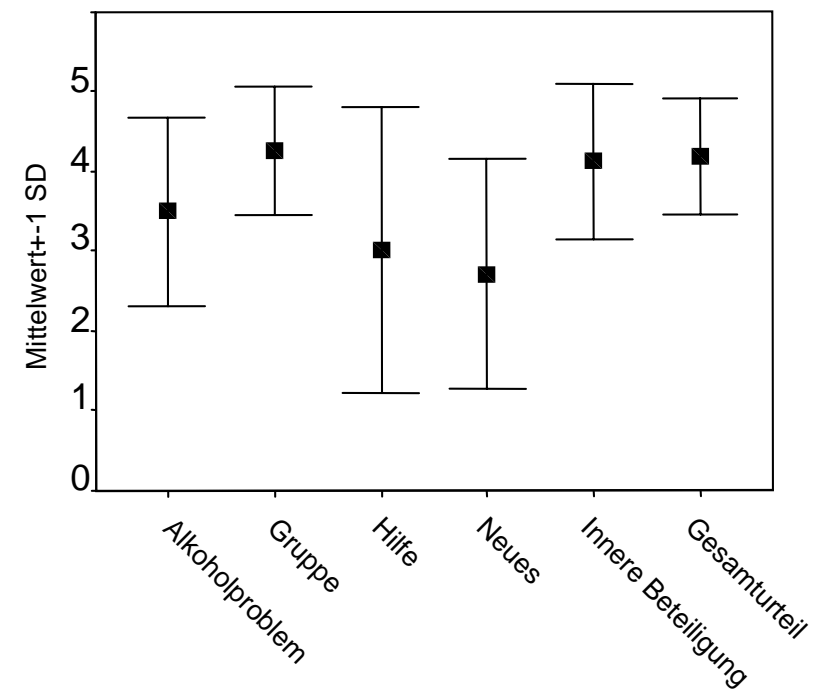

Abb. 3 Bewertung $(n=357)$ von sechs Aspekten jeder AkT-Sitzung durch die Teilnehmer (Durchschnitt \pm 1 SD über alle Gruppen und alle 10 Sitzungen). (Alkoholproblem $=$ „Heute habe ich den Eindruck, dass ich bei der Bewältigung meines Alkoholproblems vorangekommen bin“; Gruppe = „Heute habe ich mich in der Gruppe wohl gefühlt“; Hil$\mathrm{fe}=$ „Heute habe ich konkrete Hinweise und Hilfe erhalten“; Neues $=$ „Heute habe ich Dinge erfahren, die mir vorher noch nicht klar gewesen sind“; Innere Beteiligung = „Heute war ich innerlich ganz dabei“; für die ersten fünf Items: 0 = stimmt nicht, 5 = stimmt völlig; Gesamturteil = „Insgesamt betrachtet, fand ich die heutige Gruppensitzung“ [0 = sehr schlecht, 5 = sehr gut $]$ ).

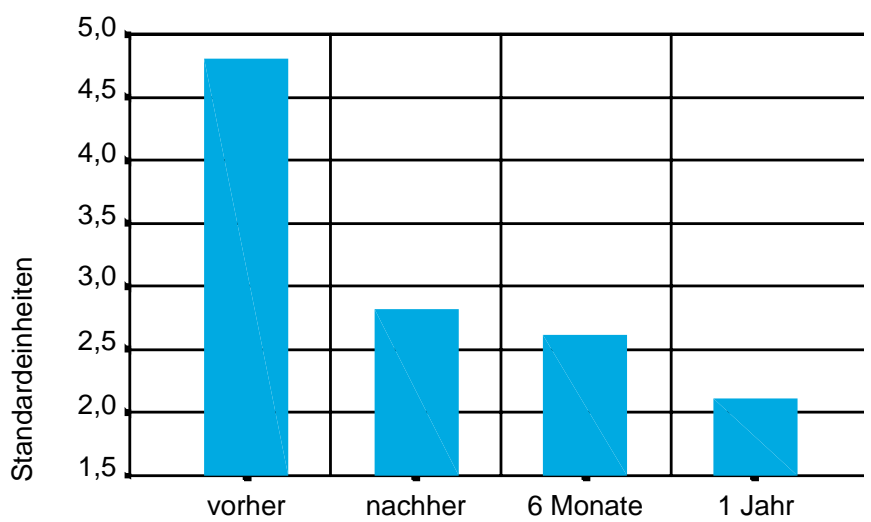

Abb. 4 Durchschnittliche tägliche Trinkmenge (in Standardeinheiten [SE]; $1 \mathrm{SE}=20 \mathrm{~g}$ Alkohol) vor und direkt nach dem AkT sowie 6 Monate und 1 Jahr nach Programmende $(n=11)$.
(14\%). Die Zielsetzung lautet bei einigen weiterhin kontrolliertes Trinken. Bei anderen konnten die Selbsterkenntnis, mit kontrolliertem Trinken überfordert zu sein und die Motivation, aktive Schritte hin zur Abstinenz zu unternehmen, gefördert werden. Alleine 5 Klienten, die vor AkT noch keinen Kontakt zum Suchthilfesystem hatten, konnten im Rahmen des AkT zur Abstinenz hingeführt werden.

\section{Folgerungen und Ausblick}

Die vorliegenden Erfahrungen mit und Befunde zum AkT sind ermutigend:

- Durch den Verzicht auf eine Abstinenzforderung sowie die überschaubare Programmdauer konnten Menschen erreicht werden, die noch nie suchtspezifische Hilfe in Anspruch genommen hatten, dieser aufgrund erheblicher Alkohol- und Alkoholfolgeprobleme aber dringend bedurften.

- Der durchschnittliche Alkoholkonsum konnte durch das AkT um mehr als die Hälfte gesenkt werden, die Anzahl abstinenter Tage nahm zu, einige Teilnehmer erreichten im Laufe des Programms vollständige Abstinenz. Diese Erfolge sind auch noch nach einem Jahr nachweisbar.

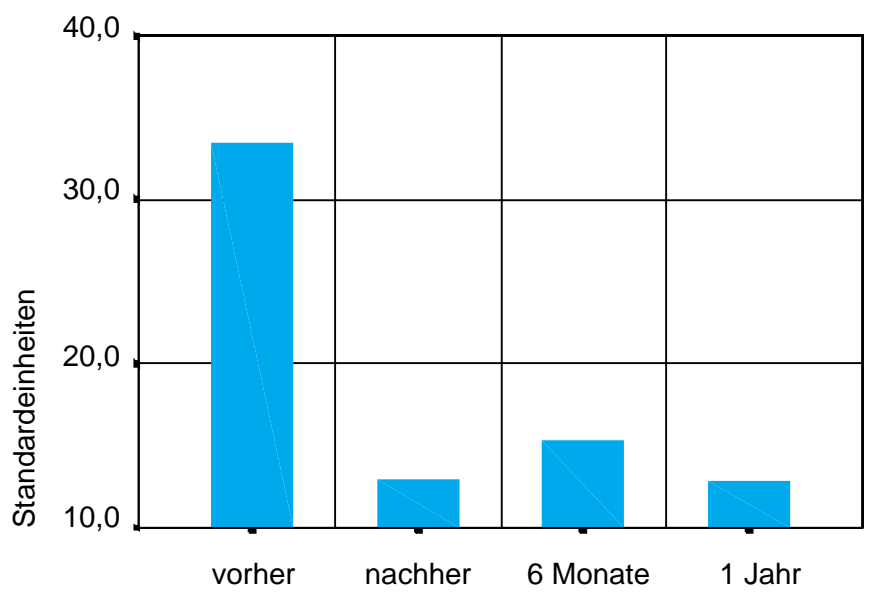

Abb. 5 Durchschnittliche wöchentliche Trinkmenge (in Standardeinheiten) vor und direkt nach dem AkT sowie 6 Monate und 1 Jahr nach Programmende $(\mathrm{n}=11)$.

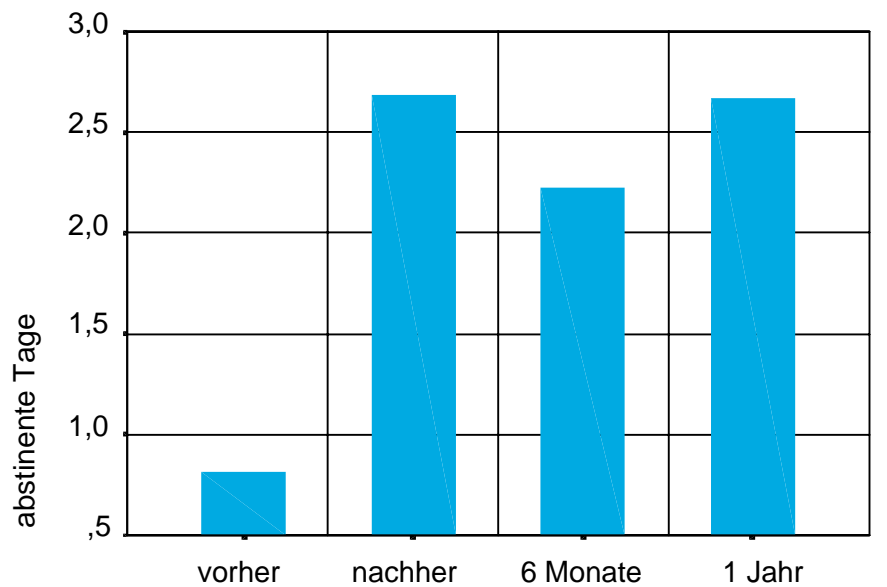

Abb. 6 Durchschnittliche Anzahl abstinenter Tage pro Woche vor und direkt nach dem AkT sowie 6 Monate und 1 Jahr nach Programmende $(n=11)$. 
- Fast die Hälfte der Teilnehmer nahm, angestoßen durch das AkT, weitere ambulante und/oder stationäre suchtspezifische Hilfe in Anspruch, so sich diese als notwendig erwies.

- Mit den Trinkmengenreduzierungen gingen Beeinträchtigungen im Lebensalltag zurück (z. B. Partnerprobleme, Leistungseinbußen am Arbeitsplatz; vgl. auch die Fallvignetten zum AkT, Beitrag Körkel et al., in diesem Heft).

- Die relativ geringe Quote klassischer Behandlungsabbrüche sowie die positiven formellen und informellen Rückmeldungen der Teilnehmer sind als weiteres Indiz für die Adäquatheit der Programminhalte und -gestaltung zu werten.

- Seit Sommer 2000 existiert eine Selbsthilfegruppe ehemaliger AkT-Teilnehmer, die sich einmal im Monat trifft. Sie begünstigt nach bisherigen Erfahrungen eine Stabilisierung der erzielten Erfolge.

Die genannten ersten Ergebnisse und begleitenden klinischen Eindrücke zum AkT sprechen für die Weiterführung und Ausweitung entsprechender Programme. Hägerbäumer [17], Bundesgeschäftsführer des Blauen Kreuz in Deutschland, folgert ganz im Sinne unserer Sichtweise: „Das AkT-Programm ist ein geeignetes Angebot, um Menschen früher zu erreichen ... Der körperliche und seelische Abbau der Suchtkranken kann früher gestoppt werden. Das Leid der Suchtkranken und Angehörigen muss nicht ausgekostet werden und manches ist eventuell vor dem Zerbrechen zu retten.“ (S. 23)

In dieses Grundverständnis adressatenorientierter, frühzeitiger Hilfe fügen sich auch die weiteren Programme zum kontrollierten Trinken ein, die inzwischen von uns entwickelt worden sind:

- Das „Ambulante Einzelprogramm zum kontrollierten Trinken (EkT)“ [4] orientiert sich am Konzept des AkT, trägt jedoch den besonderen Bedingungen der Arbeit mit Einzelklienten Rechnung. Das EkT eignet sich für Klienten, die nicht an Gruppen teilnehmen können oder wollen und für Trainer, die die Einzelarbeit bevorzugen. Auch in ländlichen Regionen, in denen es schwierig ist, eine Gruppe von nennenswertem Umfang zusammenzustellen, kann das EkT angezeigt sein.

- Das „10-Schritte-Programm zum selbstständigen Erlernen des kontrollierten Trinkens" [18] leitet in 10 aufeinander aufbauenden Schritten zur systematischen Alkoholreduktion an und kann bei auftauchenden Umsetzungsproblemen durch eine Suchtfachkraft (,10-Schritte-Coach“) unterstützt werden [19]. Das 10-Schritte Programm umfasst ca. 100 Seiten sowie einen Anhang mit Arbeits- und Informationsblättern. Zur Durcharbeitung und praktischen Umsetzung der 10 Schritte sind drei Monate einzuplanen.

- Kurz vor der Fertigstellung befindet sich ein Programm für niedergelassene Ärzte, das ein Arbeitsheft zum kontrollierten Trinken für den Patienten (mit Trink-Tagebuch, Empfehlungen zu Kontrollstrategien usw.) und eine Arztbroschüre (für Eintragungen zu Gesprächsverlauf, „Hausaufgaben“, Labor- werten usw.) zur Begleitung des Patienten enthält. Auch analoge Programme für Psychologen und betriebliche Suchtberater befinden sich vor dem Abschluss.

\section{Literatur}

${ }^{1}$ Körkel J. Das „Ambulante Gruppenprogramm zum kontrollierten Trinken“ (AkT): Grundlagen, Programmmerkmale und erste Befunde. In: Fachverband Sucht (Hrsg). Rehabilitation Suchtkranker - mehr als Psychotherapie. Geesthacht: Neuland 2001; 270-284

2 Körkel J, Langguth W, Schellberg B. Jenseits des Abstinenzdogmatismus: Das „Ambulante Gruppenprogramm zum kontrollierten Trinken“ (AkT). In: Wienberg G \& Driessen M (Hrsg). Auf dem Weg zur vergessenen Mehrheit. Innovative Konzepte für die Versorgung von Menschen mit Alkoholproblemen. Bonn: Psychiatrie-Verlag 2001; $92-116$

${ }^{3}$ GK Quest Akademie. Leitung von ambulanten Gruppenprogrammen zum kontrollierten Trinken. (Seminarausschreibung). Heidelberg: GK Quest Akademie (info@GK-Quest.de) 2002

${ }^{4}$ GK Quest Akademie. Durchführung des „Einzelprogramms zum kontrollierten Trinken (EkT)“. (Seminarausschreibung). Heidelberg: GK Quest Akademie (info@GK-Quest.de) 2002

${ }^{5}$ Körkel J, Projektgruppe kT. Diagnostik-Manual für das „Ambulante Gruppenprogramm zum kontrollierten Trinken (AkT)“. Heidelberg: GK Quest Akademie (info@gk-quest.de) 2001

${ }^{6}$ Heather N, Robertson I. Controlled Drinking (revised edition). London: Methuen 1983

${ }^{7}$ Kruse G, Körkel J, Schmalz U. Alkoholismus erkennen und behandeln. Mit literarischen Beispielen. Bonn: Psychiatrie-Verlag 2001

${ }^{8}$ Miller WR. Controlled drinking: A history and critical review. Journal of Studies on Alcohol 1983; 44: 68-83

${ }^{9}$ Rosenberg H. Prediction of controlled drinking by alcoholics and problem drinkers. Psychological Bulletin 1993; 113: 129-139

${ }^{10}$ Connors GJ, Donovan DM, DiClemente CC. Substance Abuse Treatment and the Stages of Change. Selecting and Planning Interventions. New York: Guilford 2001

${ }^{11}$ Miller WR, Rollnick S. Motivierende Gesprächsführung. Freiburg: Lambertus 1999

${ }^{12}$ Berg IK, Miller SD. Kurztherapie bei Alkoholproblemen. Ein lösungsorientierter Ansatz. Heidelberg: Carl-Auer-Systeme 1993

${ }^{13}$ Körkel J, Projektgruppe kT. Trainer-Manual für das „Ambulante Gruppenprogramm zum kontrollierten Trinken (AkT)““. Heidelberg: GK Quest Akademie (info@gk-quest.de) 2001

${ }^{14}$ Körkel J, Projektgruppe kT. Teilnehmer-Handbuch für das „Ambulante Gruppenprogramm zum kontrollierten Trinken (AkT)“. Heidelberg: GK Quest Akademie (info@gk-quest.de) 2001

${ }^{15}$ GK Quest Akademie. Aufbautraining für AkT-TrainerInnen. (Seminarausschreibung). Heidelberg: GK Quest Akademie (info@GK-Quest.de) 2002

${ }^{16}$ Körkel J. Kontrolliertes Trinken: Stand der Forschung, Folgerungen für die Praxis. In: Landesstelle gegen die Suchtgefahren für SchleswigHolstein (LSSH) (Hrsg). Kontrolliertes Trinken oder kontrollierter Rückfall. Kiel: hansadruck 2001; 15-54

${ }^{17}$ Hägerbäumer H. Aufklärung und eine veränderte Trinkkultur. PartnerMagazin 2001; 2: 19-23

${ }^{18}$ Körkel J. Das 10-Schritte-Programm zum selbstständigen Erlernen des kontrollierten Trinkens. (unter www.kontrolliertes-trinken.de) 2001

${ }^{19}$ GK Quest Akademie. 10-Schritte-Coach. (Seminarausschreibung). Heidelberg: GK Quest Akademie (info@GK-Quest.de) 2002 